\title{
Acoustic Measurement of the Length of Air-plasma Filament Induced by an Intense Femtosecond Laser Pulse
}

\author{
Si-Qing $\mathrm{WU}^{1, *}$, Bing $\mathrm{DAl}{ }^{2}$, Gang $\mathrm{XIONG}^{1}$, and Wu-Xiong $\mathrm{XU}^{1}$ \\ ${ }^{1}$ School of Electronic and Information Engineering, Hubei University of Science and technology, Xianning, 437100, China. \\ ${ }^{2}$ Wuhan National Laboratory for Optoelectronics, Huazhong University of Science and technology, Wuhan 430074, China \\ *Corresponding author: wusiqingccnu@163.com
}

\begin{abstract}
The paper studies acoustic emission from air-plasma filament induced by a strong femtosecond laser pulse. Acoustic signal is detected with a sensitive directional microphone. Acoustic measurement provides a new method to determine the length of a filament. Compared with other methods, acoustic measurement is simpler, more sensitive, and with higher spatial resolution. Information of filament length is experimentally acquired through measuring acoustic pressure at different position of filament. On the basis of the relationship between acoustic signal and free-electron density in filament, profile of free-electron density is demonstrated
\end{abstract}

\section{Introduction}

Since the advent of ultra-short laser pulses, plasma filament as a result of intense femtosecond pulse lasers propagating in air becomes a hot research field in ultra-fast spectroscopy. The intriguing potential perspectives for some physics events, such as atmospheric remote sensing[1,2], terahertz wave generation[3], lidar (light detection and ranging)[4] and lightning discharge control[5,6], attract strong interest of researchers. Experimental results reported by Brodeur[7], Nibbering[8] et al, have shown that such plasma filaments can propagate tens of meters and up to several kilometers in atmosphere, beyond Rayleigh range of laser beam[9]. The underlying physical mechanism for the optical process is considered as laser Kerr nonlinear self-focusing effect. When an intense femtosecond laser pulse propagates in air, air acts as a Kerr nonlinear medium to focus beam. In focal region, peak optical intensity increases and photoionization is induced, resulting in the generation of plasma. When dynamic equilibrium emerges between self-focusing and defocusing, a narrow and long plasma column is produced [10-12].

Because optical intensity in plasma exceeds $6 \times 10^{13}$ $\mathrm{W} / \mathrm{cm}^{2}$ [13], and electron density exceeds $10^{18} \mathrm{~cm}^{-3}$ [14], plasma filament induced by strong femtosecond laser is not directly measured using suitable instrument. Therefore, measure for plasma column is only indirectly implemented based on filament manifest phenomenon. Based on acoustic radiation from plasma induced by strong femtosecond laser pulses $[15,16]$, acoustic measuring method is employed to examine the plasma filament, depending on our laboratory existing femtosecond laser system. Length of filament is examined through detecting acoustic pressure at different position of filament.

\section{Experimental Details and Results}

In our experiment, a strong femtosecond laser pulse amplification system (Spectra-Physics) is employed to ionize air into gas plasma, which radiates photo- acoustic pulse signal in ambient. This system utilizes a mode locked Mai Tai Seed laser oscillator to generate initial pulse, and Ti: Sapphire Regenerative Amplifier is necessary to strengthen pulse energy to peak value of $5 \mathrm{~mJ}$. Output Gaussian-pulse is centered at $800 \mathrm{~nm}$ and repetition rate is set at $1 \mathrm{kHz}$. Full-width-half- magnitude (FWHM) of pulse is around $50 \mathrm{fs}$ and beam diameter is roughly $8 \mathrm{~mm}$. Laser pulse is focused into air to create plasma filament by an achromatic plano-convex spherical lens (GCL-010157B). Diameter of lens is $25.4 \mathrm{~mm}$, and its focal-length is $1000 \mathrm{~mm}$. Plasma filament is brightly visible to naked eyes in a range of about several $\mathrm{cm}$ around lens focal region.

Acoustic emission from plasma is detected by a directional microphone (HT-280) placed at a distance $\mathrm{d}$ of $2 \mathrm{~mm}$, and microphone is vertical with laser guiding direction from beginning to end. Diameter of microphone is $9 \mathrm{~mm}$, restricting the directly measured filament to a length of $14 \mathrm{~mm}$. Frequency-range of the microphone is $20 \mathrm{~Hz}-20 \mathrm{kHz}$ and sensitivity is $-38 \pm 2 \mathrm{~dB}$. Acoustic signal are transformed by microphone to an electronic signal which is then delivered to a digital phosphor oscilloscope (Tektronix DPO4032), for measurement of acoustic pulse waveform. Oscilloscope has $350 \mathrm{MHz}$ bandwidth and $1 \mathrm{M} \Omega$ input impedance. Experimental setup is presented schematically in Fig.1. 


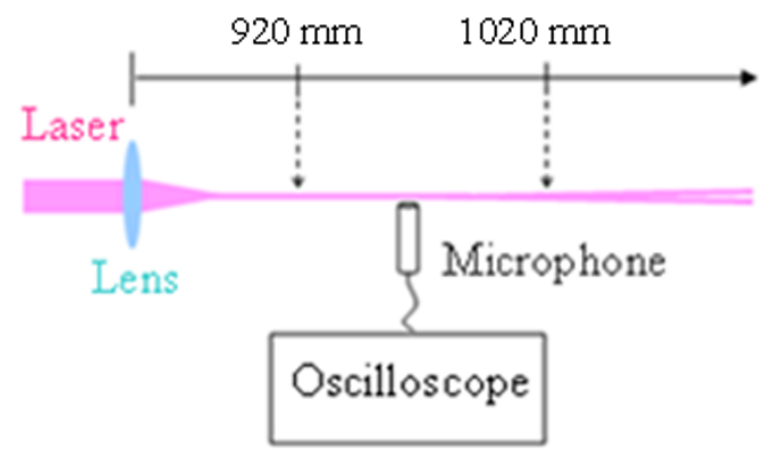

Figure1. Experimental schematic of photo acoustic emission from laser-induced air plasma filament.

By measuring amplitude of acoustic signal as a function of distance from center of lens, the dependence of the signal on propagating distance can be determined. Fig. 2 shows the results for the case of $3 \mathrm{~mJ}$ laser-pulse focused by a $1000 \mathrm{~mm}$ focal-length lens. Acoustic signal is firstly detected at the position of $920 \mathrm{~mm}$ away from center of lens. Then, the translational stage mounting directional microphone is moved along laser transporting direction with a step of $2 \mathrm{~mm}$, keeping the distance between microphone and filament unchanging, until the place standoff lens center of $1020 \mathrm{~mm}$. For reducing the influence of direction errors from microphone position, measurements in Fig. 2 are average results of several measures.

From Fig.2, one can find out that peak amplitude of acoustic signal significantly becomes stronger at the position of $928 \mathrm{~mm}$ (arrow (1)) away from center of lens, and obviously becomes smaller at the place (arrow (2)) standoff lens center of $1000 \mathrm{~mm}$. In the experiment, we also develop a thin glass piece to roughly inspect plasma filament. We find out, at about $928 \mathrm{~mm}$ location from center of lens, there is an obvious bright spot and a small pit on the thin glass piece; however, at more than $1000 \mathrm{~mm}$ position, filament cannot be examined. The experiment examining result is consistent with the changing trend of acoustic signal in Fig.2. Therefore, we can come to a conclusion that, the length of air filament induced by the focused intense femtosecond laser pulses is at least $72 \mathrm{~mm}$, from the location of $928 \mathrm{~mm}$ to the position of $1000 \mathrm{~mm}$ away from lens center. Note that, at the range of $54 \mathrm{~mm}$ from $934 \mathrm{~mm}$ (arrow (3) to $988 \mathrm{~mm}$ (arrow (4)) away from lens center, acoustic signals are relatively stronger, and we can catch sight of a bright plasma filament with naked eyes. In addition, we notice that plasma filament firstly emerges before geometrical focus of lens. The reason for this situation is mainly due to the fact that, Kerr nonlinear self-focusing effect results in laser pulses to focus ahead of time, which leads to air plasma filament forming in front of geometrical focus of lens $[15,17]$. Note that, since laser is operating at $\mathrm{kHz}$ repetition rate, measurements are thus cumulative the average of several filaments and does not primarily mimic true measurement of filament generated by single pulse.

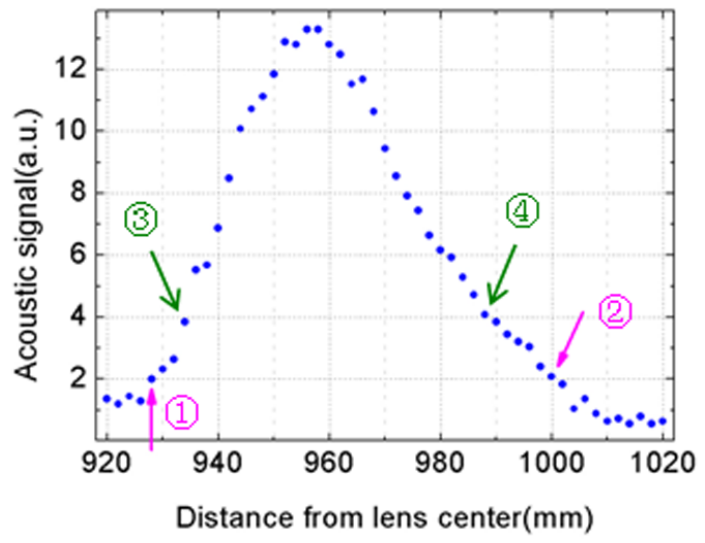

Figure.2 Peak amplitude of acoustic signal at different position.

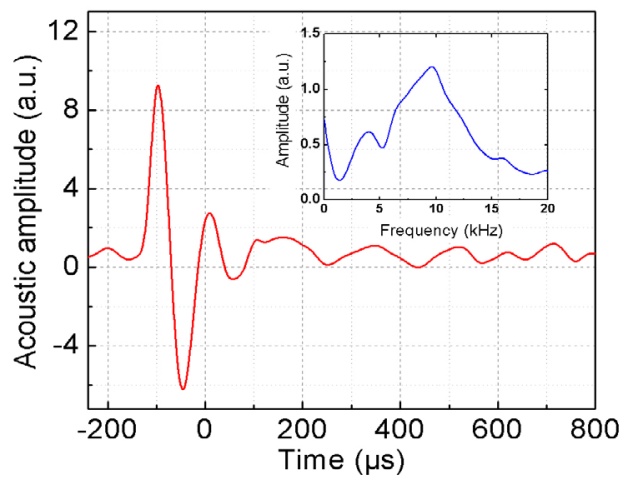

Figure.3. An acoustic signal collected by oscilloscope. Inset is corresponding spectrum.

Fig.3 shows a waveform of acoustic pulse emitted from plasma filament. The acoustic signal is recorded by directional microphone fixed at a location of $950 \mathrm{~mm}$ away from lens center, and temporally resolved using an oscilloscope with a sampling frequency of $50 \mathrm{kHz}$. This waveform is composed of $1 \sim 2$ main oscillatory pulses followed by a series of small ripples, and pulse duration is around $125 \mu \mathrm{s}$. By taking Fourier transformation, acoustic spectrum can be obtained, as showed in inset of Fig. 3 . From spectrum graph, one can understand that the spectrum covers total frequency component in microphone responding range. At the same time, we are aware of peak amplitude of acoustic pulse emerging at central frequency of microphone responding range, i.e. 10 $\mathrm{kHz}$. The dips in spectrum are a result of microphone response, and they are not related to acoustic emission. In the experiment, before and after plasma filament, we can still detect a pulsed acoustic signal, which is background acoustic noise. Background noise is measured at several $\mathrm{mv}$ in out laboratory, and noise level is 2 orders of magnitude under the maximal acoustic signal detected at the center of filament. However, background noise does not interference the measurement of acoustic signal that is drawn in Fig. 2, because noise is removed from the measured acoustic pulse.

\section{Discussion}

Under illumination of intense femtosecond laser pulses, multiphoton-ionization (MPI) of air molecules takes place 
in subpicoseond time scale through releasing free electrons, forming air plasma filament. Optical field enhances free electron temperature, Te, to the order of $10^{4}-10^{5} \mathrm{~K}$, while the temperature of neighboring molecules or ions, Tm, is much lower [18]. Within the following $10^{-9}-10^{-8} \mathrm{~s}$, Tm rapidly ascents through energy transfer due to elastic and inelastic collision between hot electrons and the surrounding air molecules (nitrogen and oxygen), until thermal equilibrium appears[19,20]. This sudden increase of localized gas temperature gives rise to a shock wave that persists for a short distance, then relaxing into acoustic wave $[21,22]$.

As to the physical processes of energy transfer between free electrons and heavy particles (ions and air molecules) in a femtosecond pulse-induced plasma, F. Vidal et al.[23] have given detailed theoretical analysis. The time of the energy transfer from electrons to heavy particles should been in the range of $10^{-9}-10^{-8} \mathrm{~s}$. The final thermal equilibrium is achieved in $10^{-6}-10^{-5} \mathrm{~s}$ after the exciting laser pulse[23], and equilibrium temperature in air filament is approximately $0.1 \mathrm{eV}$ (corresponding to a temperature of $1200 \mathrm{~K}$ ).

The relevance between acoustic pulse from air-plasma filament and initial free-electron density in air plasma was been deeply studied by some researchers. For instance, it was been verified that the maximum amplitude of acoustic signal is scaled with exciting-laser energy absorbed by the gas[24], when acoustic method was employed to survey multiphoton absorption by polyatomic molecules. In turn, the absorbed optical-energy is scaled with initial free-electron density yielded through MPI, when gas and exciting-laser wavelength are appropriate. Consequently, the detected microphone signal that is displayed in Fig. 3 provides a direct consequence for the free-electron density contour in plasma filament.

\section{Summary}

Long plasma filament can emerge as a result of strong femtosecond laser pulse focused by a large focal-length lens to propagate in air, and acoustic-pulse signal can be emitted in the ambient. Experiments show that, at different location of plasma filament, intensities of acoustic signal are discriminate, and acoustic intensities are dependent on the distance from lens center in the form of the normal distribution along laser transmission direction. Length of air plasma filament can be indirectly measured through examining acoustic signal eradiated from plasma. Being a fast, real-time and non-destructive measurement, and only easy measuring device provided, acoustic measurement is a good method for examining long plasma filament in atmosphere, in spite of some deviation arising at the position of the beginning and ending of plasma filament. Simultaneously, free-electron density profile is acquired, based on the relevance between acoustic signal and free-electron density in filament.

\section{Acknowledgement}

The authors gratefully acknowledge support from Ph.D. Foundation-Launched Project (No. BK1523) of Hubei University of Science \& Technology.

\section{References}

1. G. Mejean, J. Kasparian and E. Salmon Appl. Phys. B 77357 (2003).

2. F. Theberge, W. Liu and S. A. Hosseini Appl. Phys. B 81131 (2005).

3. B. Sun and J. Q. Yao Chinese J. Lasers 331350 (2006).

4. J. Kasparian and J. P. Wolf Opt. Commun. 152355 (1998).

5. X. M. Zhao, J. C. Diels and C. Y. Wang IEEE J. QE. 31599 (1995).

6. H. Pépin, D. Comtois and F. Vidal Phys. Plasm. 8 2532 (2001).

7. E. T. J Nibbering, P. F. Curley and G. Grillon Opt. Lett. 2162 (1996).

8. Brodeur, C. Y. Chien and F. A. Ilkov Opt. Lett. 22 304 (1997).

9. L. Wöste, C. Wedekind and H. Wille Las Optoelektron 2951 (1997).

10. S. Eisenmann, A. Pukhov and A. Zigler Phys. Rev. Lett. 98155002 (2007).

11. F. Vidal, D. Comtois and C. Y. Chien IEEE Trans. Plasma Sci. 28418 (2000).

12. W. Liu, J. Bernhardt and F. Thberge J. Appl. Phys. 102033111 (2007).

13. J. Kasparian, R. Sauerbrey and S. L. Chin. Appl. Phys. B 71877 (2000).

14. H. Yang, J. Zhang and Y. T. Li. Phys. Rev. E 66 016406 (2002).

15. Z. Q. Hao, J. Yu and J. Zhang Chin. Phys. Lett. 22 636 (2005).

16. J. Yu, D. Mondelain and J. Kasparian Appl. Opt. 42 7117 (2003).

17. S. Petit, A. Talebpour, A. Proulx and S. L. Chin Opt. Comm. 175323 (2000).

18. G. N. Gibson, R. R. Freeman and T. J. McIlrath Phys. Rev. Lett. 671230 (1991).

19. J. Yu, D. Mondelain, J. Kasparian, E. Salmon, S. Geffroy, C. Favre, et al. Appl. Opt. 427117 (2003).

20. Filin, R. Compton, D. A. Romanov and R. J. Levis Phys. Rev. Lett. 102155004 (2009).

21. X. W. Ni, B. Zou, J. P. Chen, B. M. Bian, Z. H. Shen and J. Lu Acta Phys. Sin. 7143 (1998).

22. H. Sobral, M. Villagran-Muniz, R. Navarro-Gonzalez and A. C. Raga Appl. Phys. Lett. 773158 (2000).

23. F. Vidal, D. Comtois, C. Y. Chien, A. Desparois, B. La Fontaine, $\mathrm{T} \mathrm{W}$ Johnston, et al. IEEE Trans. Plasma Sci. 28418 (2000).

24. S. L. Chin, D. K. Evans, R. D. McAlpine and W. N. Selander Appl. Phys. 21658 (1982).

25. [24]S. L. Chin, D. K. Evans, R. D. McAlpine and W. N. Selander Appl. Phys. 21658 (1982) 